\title{
RANDOM INTERVAL SCHEDULES OF REINFORCEMENT ${ }^{1}$
}

\author{
J. R. Millenson ${ }^{2}$ \\ BIRKBECK COLLEGE, UNIVERSITY OF LONDON
}

\begin{abstract}
A method for generating a reinforcement schedule that closely approximates idealized VI schedules in which reinforcement assignments occur randomly in time (RI schedules) is described. Response rates of pigeons exposed for 20 sessions to this schedule appeared very similar to response rates characteristic of arithmetic series VIs. The distribution function describing these schedules was derived and its relations to other VI distributions, as well as to FI and random ratio (RR) were shown.
\end{abstract}

In fixed interval (FI) schedules ${ }^{3}$ a reinforcement assignment occurs at the end of every $T$ sec. When the probability that each successive fixed interval will terminate with an assignment is reduced below unity but kept greater than zero, a kind of variable interval (VI) schedule results with distribution of intervals given by the geometric distribution. ${ }^{4}$ When $T$ is small this schedule approximates a random interval (RI) schedule in which reinforcements are assigned randomly in time and inter-reinforcement assignment intervals are distributed exponentially. (See Appendix below.) The RI schedule has occasionally been assumed to be an idealized version of certain VI schedules (Fleshler and Hoffman, 1962; Revusky, 1962) but its actual behavioral effects are unknown. The present study investigated its approximation, the geometric distribution, which is not difficult to generate (requiring only a probability gate and a cam timer), and some preliminary data on keypecking response rates of pigeons exposed to this schedule are reported below.

\footnotetext{
${ }^{1}$ This research was supported in part by grants from the U.S. Public Health Service and the British Medical Research Council. I am indebted to D. K. Butt of the Birkbeck College Physics Department for generous assistance in the development of the apparatus. Reprints may be obtained from the author, Birkbeck College, Malet St., W. C. 1, London, England.
}

${ }^{2}$ U. S. Public Health Service post-doctoral fellow.

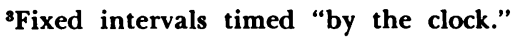

"Not to be confused with the geometric series VI of Ferster and Skinner (1957).

\section{METHOD}

\section{Subjects}

Six adult homing pigeons were used. Three birds $(35,36,37)$ had a history of 200 hr (in 16-20 hr sessions) on chained and multiple schedules, in which the key color of the present experiments had been correlated first with the initial member of a chained VI 3 FI 3 schedule, and later with the extinction component of a mult FI 3 EXT 3 schedule. The remaining three birds $(38,39,40)$ had been trained to peck a response key but were otherwise experimentally naive at the beginning of the experiment. Daily experimental sessions were scheduled unless any bird's weight was outside the limits $80 \% \pm$ $15 \mathrm{~g}$ of free-feeding weight.

\section{Apparatus}

Three similar experimental spaces for pigeons, differing only in details from the description given by Ferster and Skinner (1957), were used in this study. The response panel contained two 1 in. diameter keys, spaced 3 in. apart and symetrical to a grain magazine located below them. Each of the keys could be transilluminated independently from behind by a red lamp. Reinforcement was a 3-sec presentation of a grain mixture consisting of equal parts by weight of vetch, wheat, and hempseed. The chambers were housed in sound resistant boxes and the programming and recording apparatus were located in a separate room. A masking "white" noise source was present in the experimental room. 


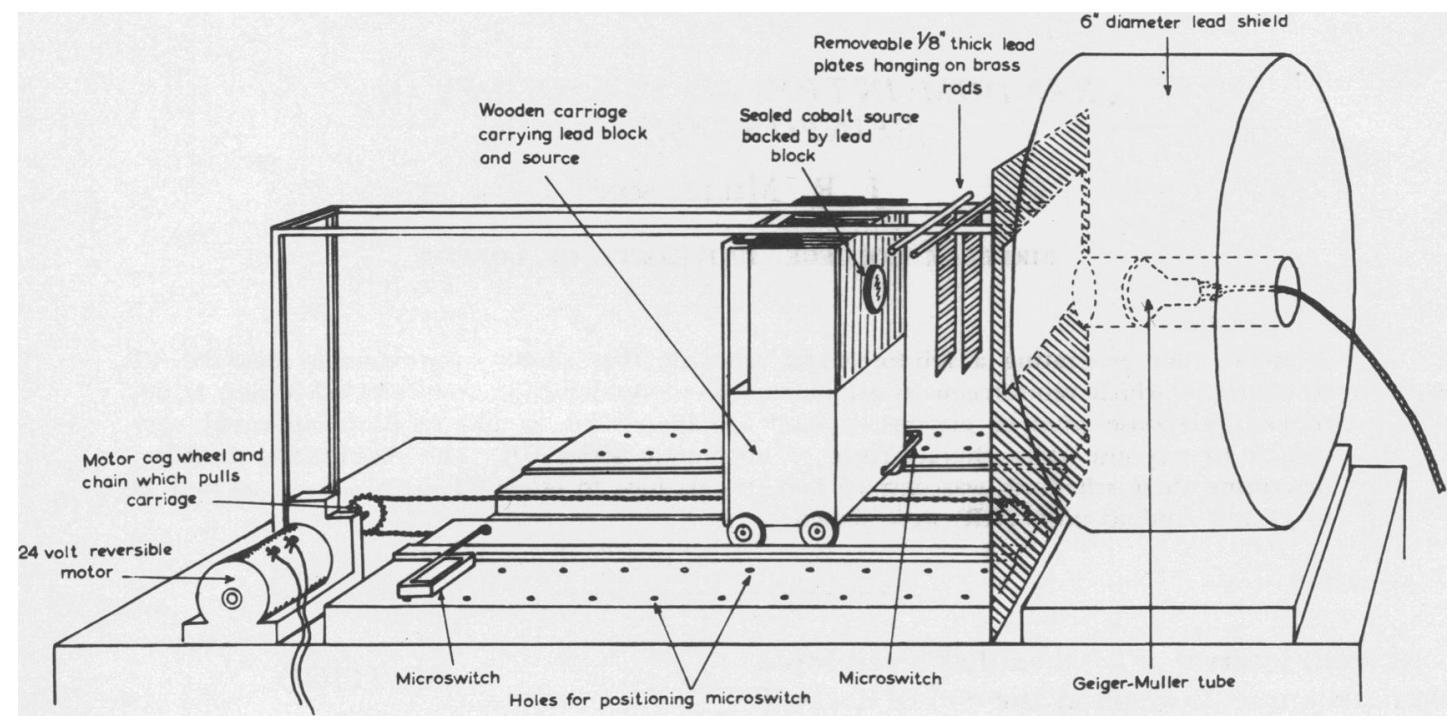

Fig. 1. Schematic diagram of the apparatus used to vary the count rate of a Geiger-Müller tube generating a random output of pulses in time.

The random interval contingencies were generated by a recycling timer in conjunction with a variable probability gate based on the natural random character of radioactive decay. This latter device, shown in Fig. 1, consisted of a lead-shielded Gieger-Müller tube and a movable Cobalt ${ }^{60}$ source imbedded in a 3 in. cube of lead. The count rate of the tube could be varied in two ways: firstly, gross adjustments could be made by the insertion of $1 / 8$ in. thick, 3 in. square fixed lead filters; secondly, by means of a reversible motor the source could be driven in a few seconds to any one of 40 positions along a 9 in. track. Excursion in either direction along the track was limited by microswitches which could be plugged in along the side of the track. The microswitch leaves intercepted the carriage, withdrawing voltage from the motor. Count pulses from the Geiger-Müller tube were amplified and

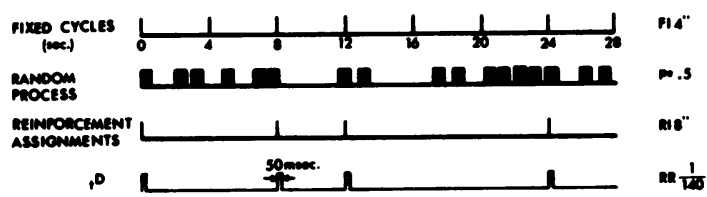

Fig. 2. Diagram illustrating the temporal relations between a fixed frequency pulse source (period $=T$ ) and a two-state random process against which it is gated. Coincidences produce an output which may be either a reinforcement assignment, or a $50 \mathrm{msec}$ period of unity probability of reinforcement for operant responses. shaped and finally actuated a plate sensitive relay which operated as a probabilistic gate, opening and closing randomly in time. The proportion of time closed was directly proportional to the rate of counting.

Electromagnetic counters, cumulative recorders, and a paper tape digital sequential event timer and recorder (SETAR) provided records of performance.

\section{Contingencies}

The top three time lines of Fig. 2 illustrate schematically the way the random interval schedules were produced. A fixed-pulse generator of constant frequency $(0.25 \mathrm{cps})$ shown in the top line is gated against a two-state random process (the Geiger gate) which, in the illustration, is in the closed state half the time on the average. Thus, the probability that any particular pulse will pass and initiate a reinforcement assignment is 0.5 . The mean random interval $(\overline{\mathrm{RI}})$ is given by: $\overline{\mathrm{RI}}=T / p$, where $T$ is the period of the constant frequency generator, and $p$ is the probability that the random process will be in the state permitting pulses to pass. In Fig. 2, $T=4 \mathrm{sec}, p=0.5$, and hence $\mathrm{RI}=8 \mathrm{sec}$. In the experiments also, $T$ was fixed at $4 \mathrm{sec}$, a value not much greater than the eating time at reinforcement; hence an important condition of VI, that occasionally successive responses were reinforced, was met. 


\section{Procedure}

With only one key illuminated, all subjects were given several sessions of approximately 60 continuous reinforcements during which the illuminated key changed sides every 10 reinforcements. Pecks on the non-illuminated key never produced reinforcement. After the first session no bird was ever observed to peck the non-illuminated key. Following this preliminary training, birds 35,36 , and 37 were run for 20 sessions on a 1 min RI (RI 1) schedule ( $T=4$ sec, $p=1 / 15)$, which contingency, along with the key color, alternated from one key to the other key every $15 \mathrm{~min}$. Birds 38 , 39 , and 40 followed a similar procedure except that a 4-min RI schedule (RI 4: $T=4$ sec, $p=1 / 60$ ) was in effect. The first three sessions for all birds were $30 \mathrm{~min}$ in length, but for the RI 1 birds the remaining sessions were $1 \mathrm{hr}$ long. For the RI 4 group, after the first three sessions, session lengths were $1 \mathrm{hr}$ long to session 10. The remaining sessions were $4 \mathrm{hr}$ long except for sessions 11 and 12, which were $19 \mathrm{hr}$ long. All sessions began with a 5-min warmup period during which response rates were not recorded, but contingencies prevailed. The particular key illuminated at the start of the session varied non-systematically from day to day.

\section{RESULTS}

\section{Random Interval Calibration}

If a schedule were to assign reinforcements truly at random in time, then the probability of an assignment in any small interval ( $t$, $t+d t$ ) would be constant, and the distribution of inter-assignment times would be exponential with a $y$ intercept equal to the average rate of assignment (Feller, 1950). The performance of our schedule programming equipment was evaluated by examining the empirical distribution of the times between successive reinforcement assignments.

The results of a typical 8-hr test, with the equipment set nominally to deliver an RI 1 are shown in Fig. 3. The points have been fitted with the exponential $\lambda e^{-\lambda^{t}}$ where $\lambda=$ average rate of assignment (estimated from these data to be 1.2 assignments per min) and $t$ is measured in 4-sec units. The expected density function for these data differs (very slightly) from the exponential due to the fact that the inter-assignment time variable is not continuous in the present schedules (see above Fig. 2 and Appendix below), but it can be seen that except for the first few points the fit is good. The systematic underestimation of the observed value at the smallest interval was genuine, occurring in all calibration curves, and is thought to be related to relative refractory times of $\mathrm{RC}$ relay pulsers associated with the probability gate. In spite of the obvious discrepancies it is apparent that over most of the range the system generates a discrete variable interval schedule closely fitted by the envelope of the theoretical continuous RI schedule matched for mean interval.

\section{Behavioral effects of RI 1 and RI 4}

The two-key procedure described above was a preparation for further work, and in the preliminary procedures studied here the schedules alternating from one key to the other every $15 \mathrm{~min}$ were identical. As no appreciable systematic rate differences were detected between the keys, the results are reported indifferently with respect to left or right key.

Cumulative records showing early (sessions 4 or 5) and late (sessions 15-20) development on the RI contingencies are shown for all birds in Fig. 4. The records for RI 1 birds 35,36 , and 37 are complete 1-hr sessions. The early sessions are shown complete for the

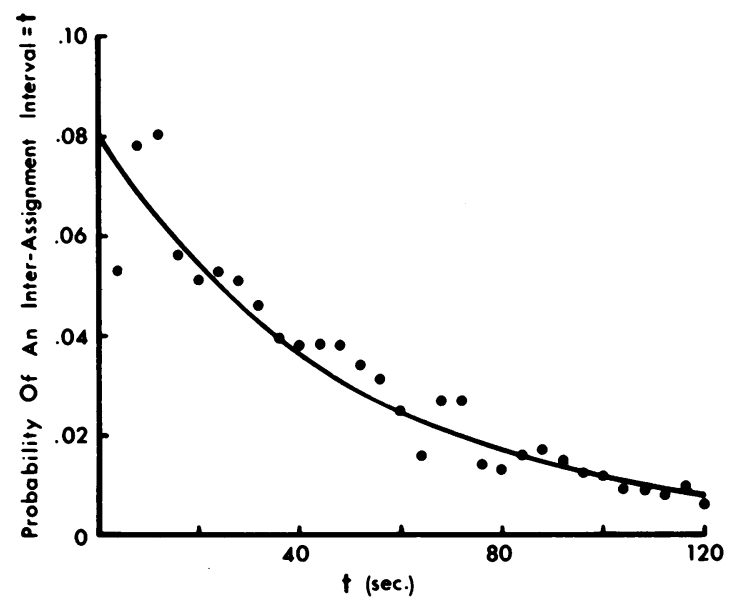

Fig. 3. Relative frequency of the distribution of inter-reinforcement assignment intervals. The points have been fitted with the exponential $\lambda e^{-\lambda_{t}} \Delta t$, where $\lambda$ is 1.2 assignments $/ \mathrm{min}, t$ is time in $4-\sec$ units, and $\Delta \mathrm{t}=4 \mathrm{sec}$. 

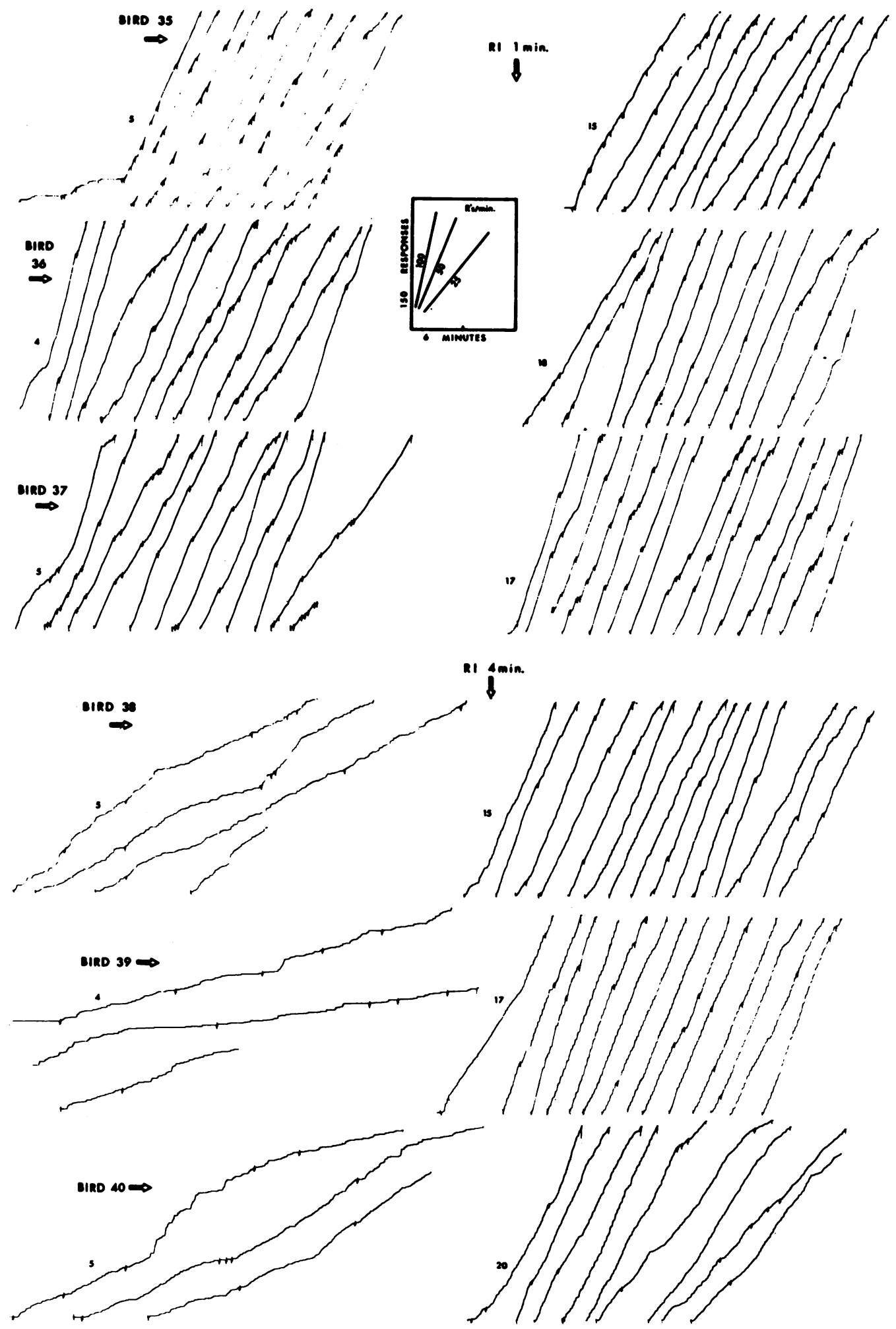

Fig. 4. Cumulative response records from six birds on RI 1 and RI 4 schedules showing two sessions for each bird. The number to the left of each set of records is the session number. 
RI 4 birds (38, 39, and 40 ), but to facilitate comparison, only the first hour of the $4-\mathrm{hr}$ long late sessions is shown.

Birds 37 and 40, run in experimental box C, showed a small but consistent pause after reinforcement which represents an extension of eating time due to grain spillage, and is not a function of the reinforcement schedules.

Comparison of the early and late sessions indicates that a development in terms of increasing overall rate and decreasing rate variability took place, similar to that reported for VI schedules (Ferster and Skinner, 1957). The transition was more rapid for the RI 1 birds, as shown by an intergroup comparison of the early sessions, but the final rates achieved by the RI 1 birds were only slightly higher than birds 38 and 39 of the RI 4 group.

Superimposed on approximately uniform overall rates, bird 39 (and to a lesser extent birds 38 and 40) on RI 4 developed a local pattern of rapid responding (bursts) followed by a short pause, during which time the bird engaged in stereotyped superstitious activity. In Fig. 4 this is best illustrated by the "wavelets" seen in session 17 of bird 39 .

The late session curves of Fig. 4 show a sustained and relatively constant overall rate, marked with occasional changes in local rate, features which characterize many VI schedules (Ferster and Skinner, 1957, p 337). Systematic pausing after reinforcement was absent and, although RI provides an occasional, very long interval, no tendency to produce negatively accelerated rates was seen.

\section{DISCUSSION}

The good agreement between the behavioral effects of the present schedules and common (arithmetic series) VI suggests that their differences have little behavioral significance. In Fig. 5 cumulative distribution functions for several VIs are compared with those of continuous RI, all with means of $2.5 \mathrm{~min}$. It will be observed that both Newman and Anger's (1954) schedule (denoted Anger, 1954) and Arithmetic Series [calculated by Findley's (1958) rule] differ somewhat from RI. Yet the present results indicate that these differences are not important enough to prevent the emergence of what are considered the cardinal features of VI performance. Fibonacci, on the other hand, is known to give rates which often are not even approximately uniform (Ferster and Skinner, p 341), and as Fig. 5 indicates, Fibonacci deviates markedly from RI both in envelope curvature, and in the large regions of zero slope (which means long periods during which no assignment is possible) at long intervals.

The present contingencies may be of more value than merely verifying the relation between VI and RI. There are, for example, several advantages that may make them useful as behavioral baselines. From a practical standpoint, they completely eliminate fixed sequence effects, and they also facilitate programming by providing a wide range of RIs merely by "dialing the $p . "$ Further, they can permit in some cases simplification of circuitry, since one RI generator often can replace many VI tape timers, e.g., when several subjects are run simultaneously on chained or tandem schedules. Secondly, an advantage is gained in specification and standardization. There exist at present almost as many VI baseline schedules as experimenters. Figure 5 hardly scratches the surface, yet only rarely is any further specification given than the mean of the interval sequence. But the mean is not always the controlling variable of the response rate in VI (Millenson, 1959). In discrete RI, there are only two parameters, $T$ and $p$, and for small $T$ s we may neglect $T$, so that the mean RI alone serves completely to denote the schedule.

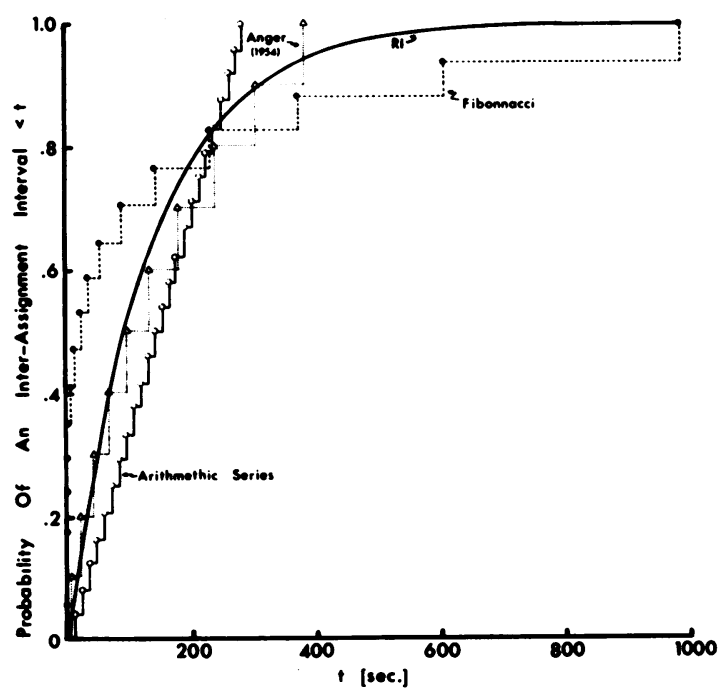

Fig. 5. Cumulative distribution functions of the inter-assignment intervals of several common VI schedules, and the exponential RI schedule. 
The merits of RI must be weighed against certain inherent drawbacks, chiefly the random deviations that any given session of RI exposure will show about the nominal mean reinforcement frequency value; and further, the necessity for installing generating and calibration equipment considerably more elaborate than the conventional tape timer of VI.

A final point which may be of some theoretical interest is the manner by which RIlocated at a junction of two independent dimensions-serves to relate FI and random ratio (RR) schedules. The relation to FI is simply that an RI is an FI in which the probability of assignment at the termination of the FI is less than 1. Interesting behavioral effects may exist where $T$ is of an order of magnitude larger than the values discussed in this paper and $p$ is close to 1 . The relation between RI and RR may be seen from the bottom time line of Fig. 2. That line describes a schedule in which, instead of initiating a reinforcement assignment, gated pulses initiate a 50 msec limited hold period during which the probability of reinforcement for a response is 1 [the $t^{D}$ of Schoenfeld, Cumming and Hearst (1956)]. Since this $t^{D}$ is smaller than the minimum IRT of the organism and comes approximately randomly in time, an approximation to Brandauer's (1958) RR schedule is produced, with the probability of reinforcement $=t^{D} / \overline{R I}$. Evidently the length of $t^{D}$ defines a continuum between $R I$ and RR whose behavioral import remains to be seen.

\section{APPENDIX}

We may define an ideal random interval schedule of reinforcement as one in which assignments would occur randomly in time at a rate $\lambda$, and the density function for interassignment times, $f(t)$ would be

$$
f(t)=\lambda e^{-\lambda t} d t
$$

To determine the analogous function for the present schedules consider the arrangement of Fig. 2. At any time $k T$ (where $k$ is any positive integer) the probability of an assignment occurring is $p$ at $k T$, and zero at all other times. Hence the probability that an interval between assignments will be exactly $n T$ long (where $n$ is a positive integer) is given by the product of the probabilities that $n-1$ previous cycles occur without an assignment, and an assignment occurs on the termination of the $n^{\text {th }}$ cycle, that is

$$
f(n T)=p \quad(1-p)^{n-1}
$$

This is the geometric distribution (c.f. Feller, 1950, $p$ 174). Note that (2) does not contain $T$ at all, and hence the form of the distribution function is independent of the size of the fixed cycle we choose. However, $T$ does enter into the moments of $f(n T)$, the mean and variance being respectively, $T / P$ and $\frac{T^{2}(1-p)}{p^{2}}$.

The geometric distribution (2) approximates the exponential distribution (1) as $T \rightarrow 0$, if the mean rate of assignment is kept fixed to a value, say, $\lambda$ (Feller, 1950).

In practice just how small must $T$ be for the approximation to be good? The exponential distribution has only one parameter, $\lambda$; furthermore, the mean and standard deviation are both $=1 / \lambda$. Matching the geometric and ideal RIs for mean, that is, letting $1 / \lambda=T / p$, we may examine the standard deviation of the former distribution as a function of $T$ to obtain an answer. In Table 1 such values appear for $1 / \lambda=R I 1$. The convergence is rapid, and at $T=4 \mathrm{sec}$ (the value used in the present study) the standard deviation differs by less than $4 \%$ from that of ideal RI. It seems unlikely that known organisms could detect a difference of this magnitude in the interval distributions, given only the cue of occasional reinforcement. From these considerations we conclude that the present contingencies closely approximate ideal $\overline{\mathbf{R I}}$, and that further reductions in $T$ (with $\overline{R I}$ held constant) would have negligible behavioral effect.

Table 1

Standard Deviations of RI 1 Schedules as a Function of $T$

\begin{tabular}{clc}
\hline$T(\mathrm{sec})$ & \multicolumn{1}{c}{$p$} & $\sigma$ \\
\hline \hline 0.05 & 0.00083 & 59.97 \\
1 & 0.017 & 59.50 \\
4 & 0.067 & 57.96 \\
16 & 0.27 & 51.38 \\
59 & 0.98 & 7.75 \\
60 & 1 & 0 \\
\hline
\end{tabular}

\section{REFERENCES}

Brandauer, C. M. The effects of uniform probabilities of reinforcement on the response rate of the pigeon. Unpublished doctoral dissertation, Columbia Univ., 1958.

Feller, W. An introduction to probability theory and its applications. (lst ed.) New York: John Wiley, 1950, Vol. 1.

Ferster, C. B. and Skinner, B. F. Schedules of reinforcement. New York: Appleton-Century-Crofts, 1957.

Findley, J. Preference and switching under concurrent scheduling. J. exp. Anal. Behav., 1958, 1, 123-144.

Fleshler, M. and Hoffman, H. S. A progression for generating variable interval schedules in which the probability of reinforcement as a function of time since reinforcement is constant. Research Bull. No. 26, Feb., 1962, Penn. State Univ. An abbreviated version of this report appears in J. exp. Anal. Behav., 1962, 5, 529-530. 
Millenson, J. R. Some behavioral effects of a twovalued temporally defined reinforcement schedule. J. exp. Anal. Behav., 1959, 2, 191-202.

Newman, E. B. and Anger, D. The effect upon simple animal behavior of different frequencies of reinforcement. Report PLR-33, Office of the Surgeon General, 1954. An abbreviated version appears as Anger, D. The dependence of interresponse times upon the relative reinforcement of different interresponse times. J. exp. Psychol., 1956, 52, 145-161.
Revusky, S. H. Mathematical analysis of the durations of reinforced inter-response times during variable interval reinforcement. Psychometrika, 1962, 27, 307-314.

Schoenfeld, W. N., Cumming, W. W., and Hearst, E. On the classification of reinforcement schedules. Proc. Nat. Acad. Sci., 1946, 42, 563-570.

Received September 10, 1962 